\title{
MAGNETIC DESIGN OF THE ADVANCED LIGHT SOURCE ELLIPTICAL WIGGLER*
}

\author{
S. Marks, J. Akre, E. Hoyer, D. Humphries, T. Jackson, \\ Y. Minamihara, P. Pipersky, D. Plate, and R. Schlueter
}

Advanced Light Source

Accelerator and Fusion Research Division

Lawrence Berkeley Laboratory

University of California

Berkeley, CA 94720

June 1995

Paper presented at the 14th International Conference on Magnetic Technology, Tampere University of Technology, Finland, June 11-16, 1995

*This work was supported by the Director, Office of Energy Research, Office of Basic Energy Sciences, Materials Sciences Division, of the U. S. Department of Energy, under Contract No. DE-AC03-76SF00098. 


\section{DISCLAIMER}

This report was prepared as an account of work sponsored by an agency of the United States Government. Neither the United States Government nor any agency thereof, nor any of their employees, make any warranty, express or implied, or assumes any legal liability or responsibility for the accuracy, completeness, or usefulness of any information, apparatus, product, or process disclosed, or represents that its use would not infringe privately owned rights. Reference herein to any specific commercial product, process, or service by trade name, trademark, manufacturer, or otherwise does not necessarily constitute or imply its endorsement, recommendation, or favoring by the United States Government or any agency thereof. The views and opinions of authors expressed herein do not necessarily state or reflect those of the United States Government or any agency thereof. 


\section{DISCLAIMER}

Portions of this document may be illegible in electronic image products. Images are produced from the best available original document. 


\section{Magnetic Design of the Advanced Light Source Elliptical Wiggler}

S. Marks, J. Akre, E. Hoyer, D. Humphries, T. Jackson, Y. Minimihara, P. Pipersky, D. Plate, R. Schlueter

Lawrence Berkeley Laboratory, I Cyclotron Road, Berkeley, California

\begin{abstract}
An elliptical wiggler has been designed for installation in the Advanced Light Source at the Lawrence Berkeley Laboratory. The design has been optimized for the production of circularly polarized light in the $50 \mathrm{eV}$ to $10 \mathrm{KeV}$ energy range. The device will be $3.4 \mathrm{~m}$ long consisting of vertical and horizontal periodic structures. The period length for both is $20 \mathrm{~cm}$. The vertical structure is a hybrid permanent magnet design which produces a peak field of $2.0 \mathrm{~T}$. The horizontal magnetic structure is an iron core electromagnetic design shifted longitudinally by one-quarter period relative to the vertical structure; it has a peak field of 0.095 , $T$. The polarity of the horizontal field can be switched at a rate of up to $1 \mathrm{~Hz}$, which results in a modulation of the chirality of the circularly polarized radiation on-axis. This paper discusses the magnetic design and presents the results of radiation spectra calculations used for determining optimal field parameter settings.
\end{abstract}

\section{INTRODUCTION}

Circularly polarized x-rays have become an important tool in the study of the spin state of magnetically ordered materials. An elliptical wiggler, designated as EW20.0, has been designed [1] for installation at the Advanced Light Source (ALS), a third generation synchrotron light source. This will provide a high flux source of elliptically polarized radiation in the $50 \mathrm{eV}$ to $10000 \mathrm{eV}$ energy range. It will be capable of switching between left and right handed polarizations at up to a $1 \mathrm{~Hz}$ rate [2]. The main parameters for EW20.0 are sumarized in Table I.

Manuscript received June 13, 1995.

This work was supported by the Director, Office of Energy Research, Office of Basic Energy Sciences, Mat. Sci. Div., of the U.S. Dept. of Energy, Contract No. DEAC03-76SF00098.
TABLE I

EW20.0 PARAMETERS

\begin{tabular}{ll}
\hline Spectral Range & $50 \mathrm{eV}-10 \mathrm{KeV}$ \\
Period Length & $20.0 \mathrm{~cm}$ \\
Number of Periods & 14 (vertical) \\
& $14 \frac{1}{2}$ (horizontal) \\
Peak Vertical Field & $2.0 \mathrm{~T}$ \\
Max. $K_{y}$ & 25.95 \\
Max. Opening Angle & $\pm 8.82 \mathrm{mrad}$ \\
Peak Horizontal Field & $0.095 \mathrm{~T}$ \\
Max. Kx & 1.5 \\
Max. Opening Angle & $\pm 0.51 \mathrm{mrad}$ \\
Max. Modulation Rate & $1 \mathrm{~Hz}$ \\
\hline
\end{tabular}

The device consists of vertical and horizontal magnetic structures. The end and longitudinal views of the magnetic configuraton are shown in Figs. 1 and 2, respectively. The vertical magnetic field is produced with a variable gap hybrid permanent magnetic structure; the horizontal field is produced with an iron core electromagnet [2]. This device is conceptually similar to one designed and built by Yamamoto, et. al., at the Photon Factory [3], except, that device uses pure permanent magnet structures for both vertical and horizontal fields, and polarization switching is accomplished by mechanically shifting the horizontal magnetic structure.

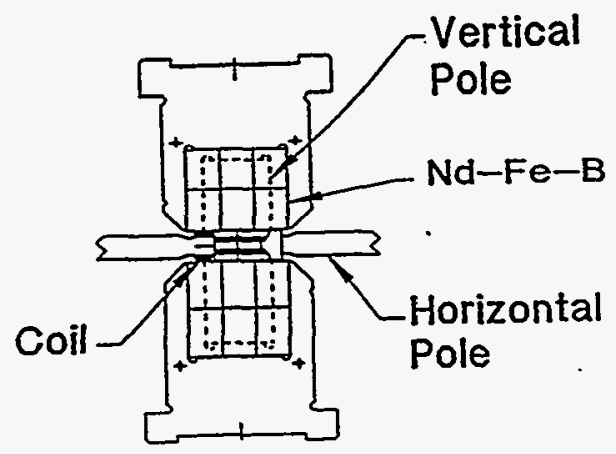

Fig. 1. EW20.0 End View of Magnetic Structure 


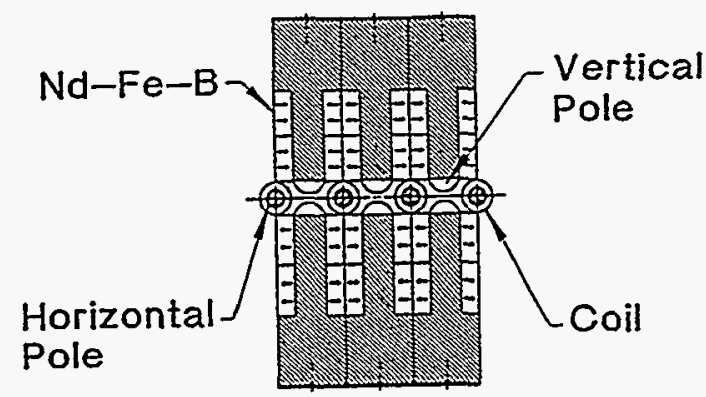

Fig. 2. EW20.0 Longitudinal View of Magnetic Structure

\section{Principle of Operation}

The production of circularly polarized light is accomplished by the mixing of the appropriately phased horizontal and vertical polarization components of a photon beam. The electric field produced by the trajectory of an electron passing through a magnetic field is the basis for radiation detected by an observer, and therefore the polarization is directly related to this motion. As an electron passes through a dipole field it bends in the plane perpendicular to the field. As viewed from directly above or below, the motion is circular, with the direction of rotation different for the two views. The apparent motion is linear as viewed from within the plane of motion. If viewed from any other angle, the apparent motion is elliptical. This qualitative behavior of apparent motion carries over to observed states of polarization. Radiation emitted from an electron passing through bend magnet is linearly polarized on axis and elliptically polarized vertically off-axis. Radiation above the axis is polarized with opposite handedness from that below the axis. This provides the basis for the use of dipole magnets as sources of elliptically polarized radiation. However, radiation from a series of wiggler poles, viewed off-axis, results in no net circular polarization, since it consists of radiation from poles of alternating magnetic polarity, and thus alternating chirality. Elliptically polarized flux can, however, be produced on the midplane through the use of a periodic horizontal magnetic field shifted $90^{\circ}$ relative to the vertical field. The horizontal field defiects the electron beam vertically so that it passes through the peak vertical field at an angle, which is equivalent to viewing successive poles first from above and then below the axis. This is schematically illustrated in Fig. 3.

The quality of radiation from an elliptical wiggler is identical to that from a bend magnet. However, it offers the advantage of higher flux due to the multiple poles.

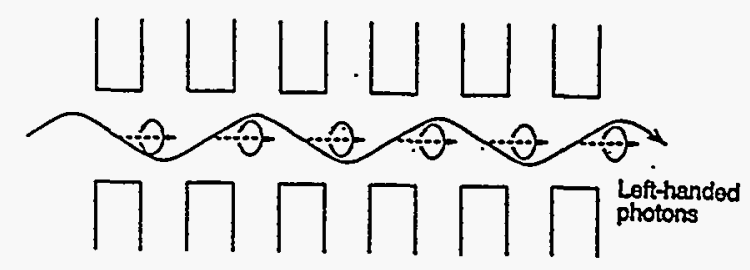

Fig. 3. Elliptical Wiggler Principle of Operation

\section{Vertical Magnetic Structure}

The vertical magnetic field structure consists of a periodic hybrid permanent magnet array with 14 periods. The peak field is $2.0 \mathrm{~T}$; this is varied by opening the gap. The pole material is vanadium permendur. The magnetic excitation is provided by $\mathrm{Nd}-\mathrm{Fe}-\mathrm{B}$ permanent magnet blocks. The poles are tapered in both the transverse and longitudinal directions to minimize saturation. The Nd-Fe-B material is recessed to accomodate the horizontal field poles. The features of the vertical structure are summarized in Table II.

TABLE II

Vertical Magnetic Structure

\begin{tabular}{ll}
\hline Magnetic Gap & $1.4 \mathrm{~cm}-22.0 \mathrm{~cm}$ \\
Peak Field & $2.0 \mathrm{~T}-0.025 \mathrm{~T}$ \\
Maximum Pole Flux Density & $2.23 \mathrm{~T}$ \\
Pole Width & $10.0 \mathrm{~cm}$ \\
Pole Thickness & $4.6 \mathrm{~cm}$ \\
Pole Height & $13.4 \mathrm{~cm}$ \\
Nd-Fe-B Operating Point & 0.52 \\
\hline
\end{tabular}

The basic building block for the periodic structure is the half-period pole assembly. This unit consists of an aluminum keeper, a pole that is pinned in the keeper and a total of twelve $\mathrm{Nd}-\mathrm{Fe}-\mathrm{B}$ blocks that are bonded to both the 
pole and the keeper, six in the front and six in the back. The half-period pole assemblies are mounted on an upper and lower backing beam. The pole assemblies are vertically aligned to within 25 microns with respect to each other on each backing beam.

The design objective for the exit and entrance region is for the average horizontal electron beam orbit through the wiggler to coincide with the central axis. This is achieved by configuring the sequence of pole scalar potentials at the entrance as $0, \frac{1}{4},-\frac{3}{4}, 1,-1$, etc., and then reversed for the exit. The amount of permanent magnet material in the end regions is adjusted so that this scalar potential sequence is maximally achieved over the full range of magnet gaps.

Tight control of integrated fields imposed by storage ring specifications will be achieved through precise alignment of the magnetic structure and by the specific arrangement of permanent magnet blocks [4]. Final trimming of integral fields will be with end coils to correct the dipole and an array of trim magnets installed in the end to correct higher order field errors [5].

\section{Horizontal Magnetic Structure}

The periodic horizontal magnetic field is produced by electromagnets. The structure has the same period length as the vertical structure, but it is shifted by one-quarter period. A total of $14 \frac{1}{2}$ periods are included to provide symmetric helicity to the electron orbit at the entrance and exit; this minimizes the production of linearly polarized light at the ends, which would decrease the total fraction of circularly polarized flux. A $0, \frac{1}{4},-\frac{3}{4}, 1,-1$ scalar potential sequence is maintained at the entrance and exit to maintain the average vertical electron orbit on the axis.

To achieve the $0.095 \mathrm{~T}$ peak field, the poles and coils are tucked in between the upper and lower vertical structures. The pole cores are laminated from $0.64 \mathrm{~mm}$ thick M36 electrical steel. A system of struts is used for support and adjustment of the poles. The peak field is varied by adjusting the current. The field polarity is switched by changing the current polarity. The coils are energized via a bipolar, regulated power supply that will provide a trapezoidal wave form for frequencies DC to $1 \mathrm{~Hz}$.

The main features of the horizontal magnetic
TABLE III

Horizontal Magnetic Structure

\begin{tabular}{ll}
\hline Peak Field & $0.0947 \mathrm{~T}$ \\
Max. Pole Flux Density & $1.96 \mathrm{~T}$ \\
Conductor & $\mathrm{Cu} 4 \mathrm{~mm} \mathrm{SQ} \times 2.5 \mathrm{~mm}$ ID \\
Number of Turns/Coil & 12 \\
Current & $500 \mathrm{~A}$ \\
Total Voltage & $135.2 \mathrm{~V}$ \\
Total Power & $66.0 \mathrm{KW}$ \\
Total Flow Rate & $25.1 \mathrm{GPM}$ \\
Coil Temp. Rise & $8.8^{\circ} \mathrm{C}$ \\
\hline
\end{tabular}

structure are summarized in Table III.

\section{RADIATION SPECTRUM}

The design objective for an elliptical wiggler is to maximize the flux of circularly polarized photons. However, the spectral output involves a trade-off between flux, $F$, and the fraction of circularly polarized photons, the degree of circular polarization, $P_{c}$. Both of these quantities are functions of the vertical and horizontal field magnitudes. In general, as the ratio of the vertical peak field to the horizontal peak field increases, the total flux increases, but the degree of circular polarization decreases. An optimization must consider a merit function which is some combination of the two quantities. The appropriate merit function for experiments using single event counting to detect asymmetry between the effects of left and right circularly polarized light is the product of the square of the degree of circular polarization and flux. We consider the integral merit function, $M$, defined as in Eq. 1 [6].

$$
M=\int_{\Delta \psi} \int_{\Delta \phi} P_{c}\left|P_{c}\right| \frac{d^{2} F}{d \phi d \psi} d \phi d \psi
$$

The horizontal and vertical angles are represented by $\phi$ and $\psi$, respectively, and $\Delta \phi$ and $\Delta \psi$ are the respective apertures.

A set of computer codes were developed to calculate the relevant spectral properties at a given photon energy for particular magnetic field parameter values and to optimize the parameter settings for maximum $M$ [7]. The results are summarized in Table IV for an electron energy of $1.5 \mathrm{GeV}$. The table includes optimum values of $B_{y}, K_{x}$, and vertical half-aperture, $\Delta \psi / 2$, and the values of optimized $M$ for a series of photon energies $\epsilon$. The results are for a fixed horizontal aperture, $\Delta \phi$, of $\pm 2.5 \mathrm{mrad}$. 
TABLE IV

OPTIMIZATION FOR $1.5 \mathrm{GEV}$

\begin{tabular}{|c|c|c|c|c|}
\hline $\begin{array}{c}\epsilon \\
\mathrm{eV}\end{array}$ & $\begin{array}{c}B_{y} \\
\text { Tesla }\end{array}$ & $\overline{K_{x}}$ & $\begin{array}{l}\Delta \psi / 2 \\
\operatorname{mrad}\end{array}$ & $\begin{array}{l}M \times 10^{-13} \\
\text { photons/sec }\end{array}$ \\
\hline 50 & 0.4 & 1.5 & 0.333 & $3.49^{\circ}$ \\
\hline 100 & 0.5 & 1.49 & 0.256 & 3.65 \\
\hline 150 & 0.5 & 1.30 & 0.229 & 3.65 \\
\hline 300 & 0.8 & 1.13 & 0.220 & 3.66 \\
\hline 700 & 1.6 & 1.07 & 0.202 & 3.59 \\
\hline 1000 & 2.0 & 1.02 & 0.191 & 3.38 \\
\hline 1500 & 2.0 & 0.88 & 0.165 & 2.94 \\
\hline 3000 & 2.0 & 0.67 & 0.127 & 1.77 \\
\hline 7000 & 2.0 & 0.48 & 0.091 & 0.415 \\
\hline 10000 & 2.0 & 0.41 & 0.079 & 0.139 \\
\hline
\end{tabular}

The results show several important trends. The optimum value of $B_{y}$ increases for increasing photon energy, in order to drive the flux intensity distribution to higher energy. Note that the peak field of $2.0 \mathrm{~T}$ is reached for a photon energy of $1 \mathrm{KeV}$. Optimized $M$ is restricted at higher photon energies because of the limit on $B_{y}$. Optimum $K_{x}$ decreases with increasing photon energy. This is because the width of the two polarization components of the radiation become narrower for higher photon energies. The optimum $M$ is limited at the lowest photon energies due to the maximum achievable $K_{x}$.

Fig. 4 shows the optimized $M$ for $1.5 \mathrm{GeV}$ and $1.9 \mathrm{GeV}$ electron energies.

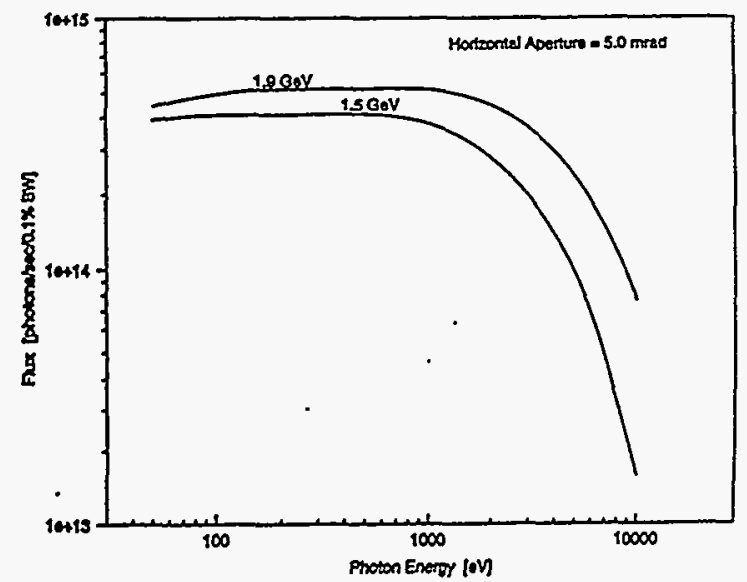

Fig. 4. Optimized $M$ for $1.5 \mathrm{GeV}$ and $1.9 \mathrm{GeV}$ electron energies. Current is $0.4 \mathrm{~A}$ and the horizontal aperture is $\pm 2.5 \mathrm{mrad}$.

\section{REFERENCES}

[1] E. Hoyer, J. Alere, D. Humphries, S. Marks, Y. Minamihara, P. Pipersky, D. Plate, and R. Schlueter, "Advanced Light Source elliptical wiggler", Rev. Sci. Instrum 66 (2), Feb., 1995.
[2] "EW20.0 Elliptial Wiggler Conceptual Design Report", LBL PUB-5400, March 1995.

[3] S. Yamamoto, T. Shioya, S. Sasaki, H. Kitamura, "Construction of insertion devices for elliptically polarized synchrotron radiation", Rev. Sci. Instrum. 60 (7), July, 1989

[4] D. Humphries, F. Goetz, P. Kownachi, S. Marks, and R. Schlueter "A multiple objective magnet sorting algorithm for the Advanced Light Source insertion devices", Rev. Sci. Instrum 66 (2), Feb., 1995.

[5] E. Hoyer, S. Marks, P. Pipersky, and R. Schlueter, "Multiple trim magnets, or 'magic fingers,' for insertion device field integral correction", Rev. Sci. Instrum 66 (2), Feb., 1995.

[6] S. Marks, W. McKinney, H. Padmore, and A. Young, "Optimizaton design study for an elliptical wiggler at the Advanced Light Source"., Rev. Sci. Instrum 66 (2), Feb., 1995.

[7] S. Marks, "Optimization Design Study for the ALS Elliptical Wiggler", LBL Light Source Beam Line Note 207, June, 1994. 